JOURNAL OF INTEGRAL EQUATIONS AND APPLICATIONS

Volume 11, Number 3, Fall 1999

\title{
CONSTRUCTIVE ANALYSIS OF PURELY INTEGRAL BOLTZMANN MODELS
}

\author{
ALVISE SOMMARIVA AND MARCO VIANELLO
}

\begin{abstract}
Existence and uniqueness of nonnegative $L^{1}$ stationary solutions of the space homogeneous force-free Boltzmann equation are proved. Classical assumptions are weakened, and certain unphysical restrictions are removed. The proof is constructive, being based on Picard iterations for an equivalent Hammerstein type formulation, within the theory of decreasing operators in ordered function spaces.
\end{abstract}

1. Introduction. Within the so-called "scattering kernel" formulation of the Boltzmann equation [4], modeling diffusion of particles in a mixture of two species (field particles and test particles), study of the space homogeneous forceless case leads naturally to the nonlinear integral equation

$$
\begin{gathered}
N \hat{g}_{r}(|\mathbf{v}|) f(\mathbf{v})+f(\mathbf{v}) \int_{\mathbf{R}^{3}} g_{r}\left(\left|\mathbf{v}-\mathbf{v}^{\prime}\right|\right) f\left(\mathbf{v}^{\prime}\right) d \mathbf{v}^{\prime}=Q_{0} S(\mathbf{v}), \\
\mathbf{v} \in \mathbf{R}^{3},
\end{gathered}
$$

which amounts to the search for stationary solutions, cf., eg., [1 $\mathbf{1}$ and references therein]. In (1) $\hat{g}_{r}$ and $g_{r}$ describe the removal effects between particles, $N$ is the fixed total density of the field particles, and $Q_{0} S(\mathbf{v})$ represents the constant and space uniform emission rate of the test particles, with $\int_{\mathbf{R}^{3}} S(\mathbf{v}) d \mathbf{v}=1$.

As pointed out in [1] , equation (1) provides a model for physical situations where removal of test particles between themselves is a dominant event, like, for instance, in the study of chemical and biological

\footnotetext{
Accepted for publication on November 25, 1998.

Key words and phrases. Space homogeneous force-free Boltzmann equation, nonnegative $L^{1}$ stationary solutions, nonlinear integral equations, Hammerstein integral equations, measurable solutions, ordered function spaces, decreasing operators, monotone approximations.

1991 AMS Mathematics Subject Classifications. 45G10, 45M20, 47H10, 47H12, $65 \mathrm{~J} 15,82 \mathrm{~B} 40$.

This work was supported, in part, by the research project, "Analisi numerica di equazioni astratte" (funds "ex 60\%," 1997-1998) of the University of Padova.

Copyright (C)1999 Rocky Mountain Mathematics Consortium
} 
action of radiations. It is worth noting that all relevant functions and parameters are positive, or at least nonnegative, due to their physical meaning; for the same reason, one is interested in a nonnegative $L^{1}$ solution, which represents the distribution of the test particles as a function of the speed. In particular, the $L^{1}$ norm of $f$ provides the unknown density of the test particles.

In the isotropic case, $S(\mathbf{v})=(4 \pi)^{-1} S_{0}(v)$, it can be seen that $f(\mathbf{v})=(4 \pi)^{-1} f_{0}(v)$ solves $(1)$, provided that $f_{0}(v)$ is a solution to

$$
\begin{gathered}
N \hat{g}_{r}(v) f(v)+f(v) \int_{0}^{\infty} G\left(v, v^{\prime}\right) f\left(v^{\prime}\right) d v^{\prime}=Q_{0} S_{0}(v), \\
v \in(0, \infty),
\end{gathered}
$$

where the kernel $G$ is given by

$$
G\left(v, v^{\prime}\right)=\frac{1}{2 v v^{\prime}} \int_{\left|v-v^{\prime}\right|}^{v+v^{\prime}} t g_{r}(t) d t .
$$

Both (1) and (2) fall into the class of nonlinear integral equations with the following quadratic structure

$$
a(x) \phi(x)+\phi(x) \int_{D} \mathcal{K}(x, t) \phi(t) d t=b(x), \quad x \in D \subseteq \mathbf{R}^{d},
$$

where $a, b: D \rightarrow \mathbf{R}^{+}$and $\mathcal{K}: D^{2} \rightarrow \mathbf{R}^{+}$are measurable, and we assume that

$$
a b>0 \quad \text { a.e. in } \quad D ; \quad b / a \in L^{1}(D) .
$$

Observe that almost everywhere positivity of $a$ and $b$ is not restrictive, provided that they do not vanish simultaneously on a positive measure subset; indeed, it is sufficient to change the domain into $\{x \in D$ : $a(x) b(x)>0\}$, since $\phi=0$ where $b=0$.

If we consider the fractional functional transformation

$$
\phi=\frac{b}{a(1+u)},
$$

equation (4) can be reformulated as

$$
u(x)=\int_{D} \frac{\mathcal{K}(x, t) b(t)}{a(x) a(t)} \frac{1}{1+u(t)} d t, \quad x \in D .
$$


The basic assumption $b(\cdot) \mathcal{K}(x, \cdot) / a(\cdot) \in L^{1}(D)$ guarantees that the integral Hammerstein problem (7) is well-defined. We stress that any nonnegative and measurable solution of (7) corresponds via (6) to a nonnegative integrable solution of (4), and conversely.

This relationship has been used in [1] to obtain existence of solutions to (2), by means of successive approximations on a Hammerstein formulation essentially equivalent to (7). The approach is classical $[\mathbf{1 4}]$ and requires strong assumptions on the kernel $G$, which are not satisfied even in very simple but physically meaningful instances, like $g_{r}(v)=c v^{p}, c>0$, with $p=-1,0,1$.

In order to circumvent this technical difficulty, in [1] equation (2) rewritten as

$$
f(v)=\frac{Q_{0} S_{0}(v)}{N \hat{g}_{r}(v)}-\frac{f(v)}{N \hat{g}_{r}(v)} \int_{0}^{\infty} G\left(v, v^{\prime}\right) f\left(v^{\prime}\right) d v^{\prime}, \quad v \in(0, \infty),
$$

has been faced directly as a fixed-point problem in the Banach space $L^{1}(0, \infty)$, by means of the contraction mapping principle. This approach allows us to include in the analysis several physically meaningful cases of removal collision frequencies $g_{r}$ and $\hat{g}_{r}$, but provides existence and uniqueness only in suitable balls of $L^{1}$, and still requires unphysical restrictions on some parameters.

A further improvement can be found in [2] where the alternative fixed-point formulation of (4)

$$
\begin{gathered}
h(x)=a(x) \phi(x)=b(x)\left(1+\int_{D} \frac{\mathcal{K}(x, t) h(t)}{a(x) a(t)} d t\right)^{-1}, \\
x \in D,
\end{gathered}
$$

has been studied in the regularly ordered Banach spaces $L^{p}(D), 1 \leq$ $p \leq \infty$, with $D$ bounded or unbounded real interval. Existence and uniqueness are there proved constructively, avoiding the constraints imposed by the contraction mapping approach, under the assumptions that $b \in L^{p}(D)$ for some $p$, there exists a constant $M>0$ such that

$$
\underset{x \in D}{\operatorname{ess} \sup } \frac{1}{a(x)}\left|\int_{D} \frac{\mathcal{K}(x, t) h(t)}{a(t)} d t\right| \leq M\|h\|_{p}
$$


for each $h \in L^{p}(D)$, and

$$
\underset{x \in D}{\operatorname{essinf}} \frac{1}{a(x)} \int_{D} \frac{\mathcal{K}(x, t) b(t)}{a(t)} d t>0 .
$$

This approach recalls previous applications to Chandrasekhar Hequation $[\mathbf{1 0}]$ and substantially relies on the fact that the nonlinear operator in (9) is decreasing on $L_{+}^{p}(D)$.

In the next section, starting from an idea of [2], we obtain existence and almost everywhere uniqueness of a nonnegative measurable solution of (7) via convergence of Picard iterations. We exploit the special decreasing structure of the Hammerstein operator, together with the key assumptions that

$$
\mathcal{K}(x, t)=\mathcal{K}(t, x) \quad \text { for a.e. } \quad(x, t) \in D^{2}, \quad b \in L^{1}(D),
$$

which is verified in the Boltzmann case, and

$$
\frac{\mathcal{K}(x, \cdot) b(\cdot)}{a(\cdot)} \in L^{1}(D), \quad \text { for a.e. } \quad x \in D,
$$

which strongly weakens (10) (put $h=b$ ) in the application to Boltzmann instances; moreover, now (11) is not required at all. Concerning (1)-(2), we stress that, differently from the contraction mapping principle, both (10)-(11) and (12)-(13) are not affected by the magnitude of the parameter $Q_{0}$.

Going back to quadratic integral equations by the transformation (6), in Section 3 we finally obtain convergence pointwise almost everywhere and in $\|\cdot\|_{1}$ of the corresponding iterations to the unique nonnegative $L^{1}$ solution. In the case of Maxwellian sources, our approach allows to treat rigorously instances of removal collision frequencies, for which only numerical investigations were feasible $[\mathbf{3}]$.

2. The Hammerstein formulation. In this section we study the following generalization of the Hammerstein integral equation (7)

$$
u(x)=\int_{D} k(x, t) g(u(t)) d t, \quad x \in D \subseteq \mathbf{R}^{d},
$$


where $k$ is measurable and almost everywhere nonnegative in $D^{2}$, the nonlinear term $g:[0,+\infty) \rightarrow(0,+\infty)$ is continuous and nonincreasing, and

(H1) the kernel $k(x, \cdot) \in L^{1}(D)$ for almost every $x \in D$, and there exists a measurable and almost everywhere positive function $\beta$ such that

$$
k(x, t) \beta(x)=k(t, x) \beta(t) \quad \text { for almost every } \quad(x, t) \in D^{2} ;
$$

(H2) the mapping $y \mapsto y g(y)$ is strictly increasing, and

$$
\beta \int_{D} k(\cdot, y) d t g\left(g(0) \int_{D} k(\cdot, y) d t\right) \in L^{1}(D) .
$$

We point out that $g>0$ is continuous nonincreasing and $y \mapsto y g(y)$ is strictly increasing in $[0,+\infty)$, if and only if

$$
g(y)=\frac{1}{c+q(y)}, \quad c>0,
$$

where $q \geq 0$ has the following properties: it is continuous and nondecreasing in $[0,+\infty)$, and it is sublinear, i.e., $q(\tau y) \geq \tau q(y)$ for every $\tau \in(0,1), y>0$. An example of such a $q$, relevant to our application, is given by $q(y)=\sum_{i=1}^{m} c_{i} y^{\alpha_{i}}$, where $c_{i} \in \mathbf{R}^{+}$, and $0<\alpha_{1}<\cdots<\alpha_{m} \leq 1$, cf. $[\mathbf{6}, \mathbf{8}, \mathbf{9}]$.

Observe that, for every almost everywhere nonnegative measurable function $u$, we have by hypothesis (H1),

$$
\begin{gathered}
\int_{D} k(x, t) g(u(t)) d t \leq \int_{D} k(x, t) g(0) d t<\infty \\
\text { for a.e. } \quad x \in D
\end{gathered}
$$

so that it makes sense to look for a nonnegative solution of (14) in any subspace of $\mathcal{M}(D)$, the quotient space of measurable functions modulo almost everywhere equivalence. We also stress that a usual assumption in similar frameworks, cf., $[\mathbf{6}, \mathbf{8}, \mathbf{1 3}]$, i.e.,

$$
\underset{x \in D}{\operatorname{ess} \sup } \int_{D} k(x, t) d t<\infty
$$


is now dropped and substituted by (H1), which allows an infinite supremum.

\subsection{Existence and uniqueness of a measurable solution.} The quotient space $\mathcal{M}(D)$ is naturally endowed with the pointwise almost everywhere convergence, and can be partially ordered by the closed cone $\mathcal{M}_{+}(D)=\{u \in \mathcal{M}(D): u(x) \geq 0$ a.e. $\}$, i.e., $v \succeq u$ if $v-u \in \mathcal{M}_{+}(D)$. Such a cone is regular, i.e., any monotone and bounded in order sequence in $\mathcal{M}_{+}(D)$ has a limit. Clearly the cone is also normal, in the sense that the "two militia-men theorem" holds $[\mathbf{9}$, 11]. The operator $A: \mathcal{M}_{+}(D) \rightarrow \mathcal{M}_{+}(D)$ defined by

$$
A(u)(x)=\int_{D} k(x, t) g(u(t)) d t, \quad x \in D
$$

is decreasing, i.e., $u \preceq v$ implies $A(u) \succeq A(v)$.

We are now ready to state and prove our main result about solvability and uniqueness for the present class of Hammerstein integral equations.

Theorem 2.1. Assume that (H1) and (H2) hold. Then the Hammerstein integral equation (14) has a unique solution in $\mathcal{M}_{+}(D)$ which is the pointwise almost everywhere limit of Picard iterations, $u_{n+1}=$ $A\left(u_{n}\right), n=0,1,2, \ldots$, starting from any $u_{0} \in \mathcal{M}_{+}(D)$.

Proof. If we consider the Picard iterations $u_{n+1}=A\left(u_{n}\right), u_{0}=$ $\theta$ (the zero element of $\mathcal{M}(D)$ ), by a classical reasoning within the theory of decreasing operators in abstract cones [9, Section 2.1], it is straightforward to prove that

$$
\theta=u_{0} \preceq u_{2} \preceq \cdots \preceq u_{2 n} \preceq u_{2 n+1} \preceq \cdots \preceq u_{3} \preceq u_{1}=A(\theta) .
$$

Moreover, there exist in $\mathcal{M}_{+}(D)$ the pointwise limits

$$
\theta \preceq \lim u_{2 n}=u^{*} \preceq v^{*}=\lim u_{2 n+1} \preceq A(\theta),
$$

and, by the dominated convergence theorem,

$$
u^{*}=A\left(v^{*}\right), \quad v^{*}=A\left(u^{*}\right) .
$$


We shall prove below that (H1) and (H2) imply the existence result $u^{*}=v^{*}$; the procedure is very close to that used in [12], where a pointwise approach allows one to work in general (nonnormed) ordered functional spaces. From the latter result uniqueness of a nonnegative measurable fixed-point immediately follows: in fact, if $\xi=A(\xi) \in \mathcal{M}_{+}(D)$, inductively one proves $u_{2 n} \preceq \xi \preceq u_{2 n+1}$ for every $n$, wherefrom $\xi=u^{*}$. Similarly it can be shown that Picard iterations converge to the unique nonnegative fixed-point, starting from any $u_{0} \in \mathcal{M}_{+}(D)$, cf. $[\mathbf{9}$, Theorem 2.1.5].

When (H1) and (H2) hold, starting from (23) we write

$$
\begin{aligned}
& \beta(x) u^{*}(x) g\left(u^{*}(x)\right)=\beta(x) g\left(u^{*}(x)\right) \int_{D} k(x, t) g\left(v^{*}(t)\right) d t, \\
& \beta(x) v^{*}(x) g\left(v^{*}(x)\right)=\beta(x) g\left(v^{*}(x)\right) \int_{D} k(x, t) g\left(u^{*}(t)\right) d t .
\end{aligned}
$$

The lefthand sides of (24) are both in $L^{1}(D)$ in view of (H2) and (22); indeed, $A(\theta)=g(0) \int_{D} k(\cdot, t) d t$ and $\beta A(\theta) g(A(\theta)) \in L^{1}(D)$ by (16). Integrating on both sides of (24) and subtracting, we get by Fubini theorem and symmetry of $k(x, t) \beta(x)$

$$
\int_{D} \beta(x)\left[v^{*}(x) g\left(v^{*}(x)\right)-u^{*}(x) g\left(u^{*}(x)\right)\right] d x=0 .
$$

Now, almost everywhere positivity of $\beta$ entails that $v^{*}(x) g\left(v^{*}(x)\right)=$ $u^{*}(x) g\left(u^{*}(x)\right)$ for almost every $x \in D$, and thus, in view of the strict monotonicity of $y g(y), u^{*}=v^{*}$ in $M_{+}(D)$.

Remark 2.2. We stress that the existence result $u^{*}=v^{*}$ is equivalent to nonexistence of distinct and comparable coupled fixed-points of $A$ in $\mathcal{M}^{+}(D)$. In fact, if $\xi_{1}, \xi_{2} \in \mathcal{M}_{+}(D), \xi_{1}=A\left(\xi_{2}\right), \xi_{2}=A\left(\xi_{1}\right)$, and $\xi_{1} \preceq \xi_{2}$, then by induction we get $u_{2 n} \preceq \xi_{1} \preceq \xi_{2} \preceq u_{2 n+1}$, and thus $u^{*}=\xi_{1}=\xi_{2}=v^{*}$.

Remark 2.3. When $D$ is bounded, convergence is almost uniform, in view of the Severini-Egoroff theorem [7, Chapter 2]. The same observation applies if $k \in L^{1}\left(D^{2}\right)$, which also ensures (dominated) convergence in $L^{1}(D)$. 
Remark 2.4. In many applications it is important to know whether the solution is continuous in $D$. This is ensured whenever

$$
\lim _{x \rightarrow x_{0}} \int_{D}\left|k(x, t)-k\left(x_{0}, t\right)\right| d t=0, \quad x_{0} \in D,
$$

by the regularizing effect of the integral operator. This also gives continuity of the Picard approximations, together with pointwise everywhere convergence to the fixed-point, and even uniform convergence on compact subsets of $D$ (by Dini's theorem [5, Theorem 7.2.2]).

3. Application to particle transport theory. The results of Section 2 are applicable to the Hammerstein formulation (7) of the quadratic integral equation (4), via the transformation (6). We state the ensuing solvability and uniqueness for (4) as a corollary of Theorem 2.1; this corollary will then be applied to the Boltzmann models (1)-(2).

Corollary 3.1. Assume that (5), (12) and (13) hold. Then the quadratic integral equation (4) has a unique solution in $L_{+}^{1}(D)$, say $\phi^{*}$, which the limit, pointwise almost everywhere and in $\|\cdot\|_{1}$ of the sequence

$$
\phi_{n+1}(x)=\frac{b(x)}{a(x)+\int_{D} \mathcal{K}(x, t) \phi_{n}(t) d t}, \quad n=0,1,2, \ldots
$$

starting from any $\phi_{0}$ such that $0 \leq \phi_{0}(x) \leq b(x) / a(x)$ for almost every $x \in D$.

When $a, b \in C(D), a(x)>0$ for every $x \in D$ and

$$
\lim _{x \rightarrow x_{0}} \int_{D}\left|\mathcal{K}(x, t)-\mathcal{K}\left(x_{0}, t\right)\right| \frac{b(t)}{a(t)} d t=0, \quad x_{0} \in D,
$$

then $\phi^{*}$ and $\phi_{n}$ are continuous in $D$, and $\phi_{n}$ converges to $\phi^{*}$ uniformly on compact subsets of $D$.

Remark 3.2. Observe that (28) is certainly satisfied if $\mathcal{K}(x, t)$ is continuous at every $x_{0}$ for almost every $t \in D$, and $\mathcal{K}(x, t) b(t) / a(t)$ is locally bounded in $x$ by an integrable function, i.e., there exist 
$\delta_{0}>0$ and $h_{0} \in L^{1}(D)$ such that $\mathcal{K}(x, t) b(t) / a(t) \leq h_{0}(t)$ for all $x \in B\left[x_{0}, \delta_{0}\right] \cap D$ and for almost every $t \in D$. In fact, in this case we are entitle to take the limit under the integral symbol, cf. $[\mathbf{7}$, Theorem $2.27]$.

Proof of Corollary 3.1. Identifying the kernel and the decreasing nonlinearity in the Hammerstein formulation (7) as

$$
k(x, t)=\frac{\mathcal{K}(x, t) b(t)}{a(x) a(t)}, \quad g(y)=\frac{1}{1+y},
$$

assumption (H1) of Theorem 2.1 is immediately verified, as by (5) and (13) we have that $k(x, \cdot) \in L^{1}(D)$, and by symmetry of $\mathcal{K}$, cf. (12), property (15) holds choosing the "symmetrizer" $\beta=b$. Moreover, (H2) is trivially satisfied, since $\beta=b \in L^{1}(D)$ by (12), and $y \mapsto y g(y)$ is strictly increasing and bounded.

Thus we are entitled to assert that equation (7), under assumptions (12)-(13), has a unique solution in $\mathcal{M}_{+}(D)$, say $u^{*}$, which is the pointwise almost everywhere limit of Picard iterations, starting from any $u_{0} \in \mathcal{M}_{+}(D)$. Applying the transformation (6), $\phi=b /(a(1+u))$, we obtain that the quadratic integral equation (4)-(5) has a unique solution in $L_{+}^{1}(D)$,

$$
\theta \preceq \phi^{*}=\frac{b}{a\left(1+u^{*}\right)} \preceq \frac{b}{a} \in L^{1}(D),
$$

which is the pointwise almost everywhere limit of the transformed sequence $\phi_{n}=b /\left(a\left(1+u_{n}\right)\right)$, cf. (27); convergence holds also in $\|\cdot\|_{1}$, being dominated by $b / a$. In fact, as already observed in the introduction, any nonnegative and measurable solution of (7) corresponds via (6) to a nonnegative integrable solution of (4), and conversely, because if $\phi \succeq \theta$ solves (4) then, necessarily, $\phi \preceq b / a$.

Remark 3.2 ensures that the Picard iterations $u_{n}$, as well as the solution $u^{*}$, are continuous in $D$, whenever (26) holds; in such a case convergence is uniform on compact subsets of $D$. Here (26) is implied by (28) when $a$ is continuous and everywhere positive in $D$, since $1 / a$ turns out to be continuous, cf. (5). Thus, when (28) holds, $a, b \in C(D)$, and $a(x)>0$ for every $x \in D$, we have that $\phi^{*}$ and $\phi_{n}$ are continuous, 
and $\phi_{n}$ converges to $\phi^{*}$ pointwise everywhere in D. Again, such a convergence becomes uniform on compact subsets of $D$, since

$$
\left|\phi_{n}(x)-\phi^{*}(x)\right| \leq \frac{b(x)}{a(x)}\left|u_{n}(x)-u^{*}(x)\right|, \quad x \in D,
$$

and $b / a$ is locally bounded in $D$, being continuous there.

We'll now check the validity of (5) and (13) in some instances of the functions $g_{r}, \hat{g}_{r}$ and $S$, characterizing the Boltzmann models (1)-(2), as special cases of (4). Note that (12) is certainly satisfied, since the kernels are symmetric and the sources are integrable, in view of their physical meaning.

Concerning (1), consider the case of a concentrated source like $S(\mathbf{v})=c|\mathbf{v}| \chi_{[-1,1]^{3}}(\mathbf{v}), c^{-1}=\int_{[-1,1]^{3}}\left|v^{\prime}\right| d \mathbf{v}^{\prime}=4 / 3$, and of constant cross section for the removal frequencies, i.e., $\hat{g}_{r}=g_{r}=i d$, cf. $[\mathbf{3}]$. Assumptions (5) and (13) trivially hold for $D=[-1,1]^{3}$, while (10) does not, since

$$
\frac{1}{|\mathbf{v}|} \int_{[-1,1]^{3}} \frac{S\left(\mathbf{v}^{\prime}\right)}{\left|\mathbf{v}^{\prime}\right|}\left|\mathbf{v}-\mathbf{v}^{\prime}\right| d \mathbf{v}^{\prime} \longrightarrow \infty, \quad|\mathbf{v}| \rightarrow 0
$$

where we put $h=b\left(=Q_{0} S\right)$ in (10). This simple example makes it clear that (13) is much weaker than (10), as pointed out in the introduction.

Following [3], in the isotropic case (2) we consider a Maxwellian source

$$
S_{0}(v)=4 \pi^{-1 / 2} \mu^{3 / 2} v^{2} e^{-\mu v^{2}},
$$

with $\mu$ positive constant proportional to the mass of a test particle, and removal collision frequencies of the form

$$
\begin{gathered}
\hat{g}_{r}(v)=\hat{c} v^{l}, \quad g_{r}(v)=c v^{q}, \\
l, q=-2,-1,0,1,2, \quad c, \hat{c}>0
\end{gathered}
$$

which ensures the validity of (5). We get immediately from (3)

$$
G\left(v, v^{\prime}\right)= \begin{cases}c\left(\left(v+v^{\prime}\right)^{q+2}-\left|v-v^{\prime}\right|^{q+2}\right) /\left(2(q+2) v v^{\prime}\right) & \text { if } q \neq-2 \\ c\left(\log \left(v+v^{\prime}\right)-\log \left(\left|v-v^{\prime}\right|\right)\right) / 2 v v^{\prime} & \text { if } q=-2\end{cases}
$$


It is easily seen that $\left(v^{\prime}\right)^{2-l} G\left(v, v^{\prime}\right) \in L_{v^{\prime}}^{1}(0, v)$ for every pair $(l, q)$, and thus (13) is satisfied; hence, the first part of Corollary 3.1 applies. Moreover, when $q \neq-2$, it can be checked that, for every $v_{0}>0$ and for every $\delta \in\left(0, v_{0}\right)$, there exist $\alpha=\alpha\left(v_{0}, \delta\right)$ and $\beta=\beta\left(v_{0}, \delta\right)$, such that the following estimate

$$
\begin{gathered}
G\left(v, v^{\prime}\right) \leq \alpha \chi_{\left(0, v_{0}+\delta\right]}\left(v^{\prime}\right)+\beta\left(v^{\prime}\right)^{q} \chi_{\left(v_{0}+\delta, \infty\right)}\left(v^{\prime}\right) \\
v \in\left[v_{0}-\delta, v_{0}+\delta\right], \quad v^{\prime}>0
\end{gathered}
$$

holds. In deriving (36), we have considered three cases, namely, $0<v^{\prime}<v_{0}-\delta \leq v, v^{\prime}>v_{0}+\delta \geq v$, and $v^{\prime} \in\left[v_{0}-\delta, v_{0}+\delta\right]$; in the latter, $G\left(v, v^{\prime}\right)$ is bounded, being continuous in $(0, \infty) \times(0, \infty)$. We omit, for brevity, the rest of the easy but tedious check. Now Remark 3.2 ensures that (28) is satisfied, and thus the solution of (2) is continuous in $(0, \infty)$, and it is the uniform limit of the Picard iterations on compact subsets of $(0, \infty)$.

On the other hand, for some pairs $(l, q)$, the results in $[2]$ cannot be applied. In fact, for $q \leq 0$, and $l>0$, it is not difficult to show that

$$
\lim _{v \rightarrow 0^{+}} \frac{1}{v^{l}} \int_{0}^{\infty} G\left(v, v^{\prime}\right)\left(v^{\prime}\right)^{2-l} e^{-\mu\left(v^{\prime}\right)^{2}} d v^{\prime}=\infty
$$

i.e., (10) does not hold (put $h=b=Q_{0} S_{0}$ ). Summarizing, we have shown that the method proposed in $[\mathbf{3}]$ converges pointwise almost everywhere and in $L^{1}(0, \infty)$, to the unique positive solution of (2)-(33)-(34), with no need of artificial restrictions.

Acknowledgments. The authors wish to thank the referees, whose observations have led to a clarification of the results and to a substantial improvement of the paper.

\section{REFERENCES}

1. V.C. Boffi and G. Spiga, An equation of Hammerstein type arising in particle transport theory, J. Math. Phys. 24 (1983), 1625-1629.

2. V.C. Boffi, R.L. Bowden and G. Spiga, On the solutions to a class of nonlinear integral equations arising in transport theory, J. Math. Phys. 25 (1984), 3444-3450.

3. V.C. Boffi, G. Spiga and J.R. Thomas, Jr., Solution of a nonlinear integral equation arising in particle transport theory, J. Comput. Phys. 59 (1985), 96-107. 
4. C. Cercignani, The Boltzmann equation and its applications, Springer, New York, 1988.

5. J. Dieudonné, Foundations of modern analysis, Academic Press, New York, 1969.

6. L. Erbe, D. Guo and X. Liu, Positive solutions of a class of nonlinear integral equations and applications, J. Integral Equations Appl. 4 (1992), 179-195.

7. G.B. Folland, Real analysis, John Wiley and Sons, New York, 1984.

8. D. Guo, Positive fixed points and eigenvectors of noncompact decreasing operators with applications to nonlinear integral equations, Chin. Ann. Math. 4 (1993), 419-426.

9. D. Guo and V. Lakshmikantham, Nonlinear problems in abstract cones, Academic Press, Inc., New York, 1988.

10. R.W. Leggett, $A$ new approach the $H$-equation of Chandrasekhar, SIAM J. Math. Anal. 7 (1976), 542-550.

11. M.A. Krasnoselskii, Positive solutions of operator equations, P. Noordhoff, Groningen, 1964.

12. A. Sommariva and M. Vianello, Approximating fixed-points of decreasing operators in spaces of continuous functions, Numer. Funct. Anal. Optim. 19 (1998), 635-646.

13. - Constructive approximation for a class of perturbed Hammerstein integral equations, Nonlinear Anal., in press.

14. F.G. Tricomi, Integral equations, Interscience, New York, 1957.

Dipartimento di Matematica Pura e Applicata, Università di Padova, Via Belzoni 7, 35131 Padova, Italy

E-mail address: alvise@brouwer.math.unipd.it

Dipartimento di Matematica Pura e Applicata, Università di Padova, Via Belzoni 7, 35131 Padova, Italy

E-mail address: marcov@brouwer.math.unipd.it 